\title{
Earth Resources Observation and Science Center- Keeping Watch Over Earth's Resources
}

The Earth Resources Observation and Science (EROS) Center is the largest facility of its kind within the U.S. Geological Survey (USGS). As both a science and data center, EROS serves a unique and critical role in shaping our understanding of a changing planet.

EROS opened its doors in 1973 as a receiving station, data archive, and data distribution hub for the USGS Landsat series of Earth observing satellites. In the nearly five decades since, EROS has grown into a globally recognized leader in land change science and a flagship information hub for the Department of Interior.

As of mid-2021, the growing EROS archive contained 65 petabytes (65 million gigabytes) of data, including data from Landsat and European Space Agency (ESA) Sentinel-2 satellites, National Aeronautics and Space Administration (NASA) satellites like Terra and Aqua, imagery from aerial and unmanned aircraft systems (drones), historical aerial photography dating back to the 1930s, and light detection and ranging (lidar) data from cutting-edge sensors like the Global Ecosystem Dynamics Investigation (GEDI) instrument on board the International Space Station.

Every individual contributes to the USGS and EROS missions. Scientists at EROS use high-performance computing, artificial intelligence, and machine learning techniques to produce detailed land cover and land change maps that serve to further scientific inquiry around the Nation and the world, as well as to aid emergency response, land and water resource management, urban planning and resiliency, carbon sequestration efforts, and ecosystem health and restoration, among others. Engineers and data technicians, meanwhile, work to ensure Landsat data quality and continuity and to maintain access to the publicly funded data resources contained within the archive.

Each branch of EROS furthers the mission of documenting, understanding, and responding to land change across the globe.

Large satellite dish on the front lawn of the EROS Center.

\section{Satellite and Ground Systems Operations}

EROS serves as the ground systems headquarters for Landsat, where data are collected, corrected, stored, and shared worldwide. Moving the data from the satellite to the public record is a complex task that involves interagency coordination and leans on multiple international partnerships.

EROS is one of several receiving stations for raw Landsat data, ingested as the satellites pass over the center of the conterminous United States. The Ground Operations Team at EROS manages the receiving antenna and supports 5-6 daytime and 5-6 nighttime data collects each day, 7 days a week. EROS also receives data from the international ground system network, including ground stations in Svalbard, Norway; Neustrelitz, Germany; Alice Springs, Australia; and Fairbanks, Alaska.

Raw Landsat data are processed at EROS, where geometric and radiometric corrections are applied to ensure that data collected at that moment align with the correct points on the Earth's surface and with data collected by previous Landsat satellites back to 1972. The EROS Calibration/ Validation Center of Excellence (ECCOE) serves as an international leader in calibration expertise, collaborating with other agencies to help improve Landsat's accuracy and reliability, and to aid efforts to harmonize Landsat data for use alongside data from similar satellite sources.

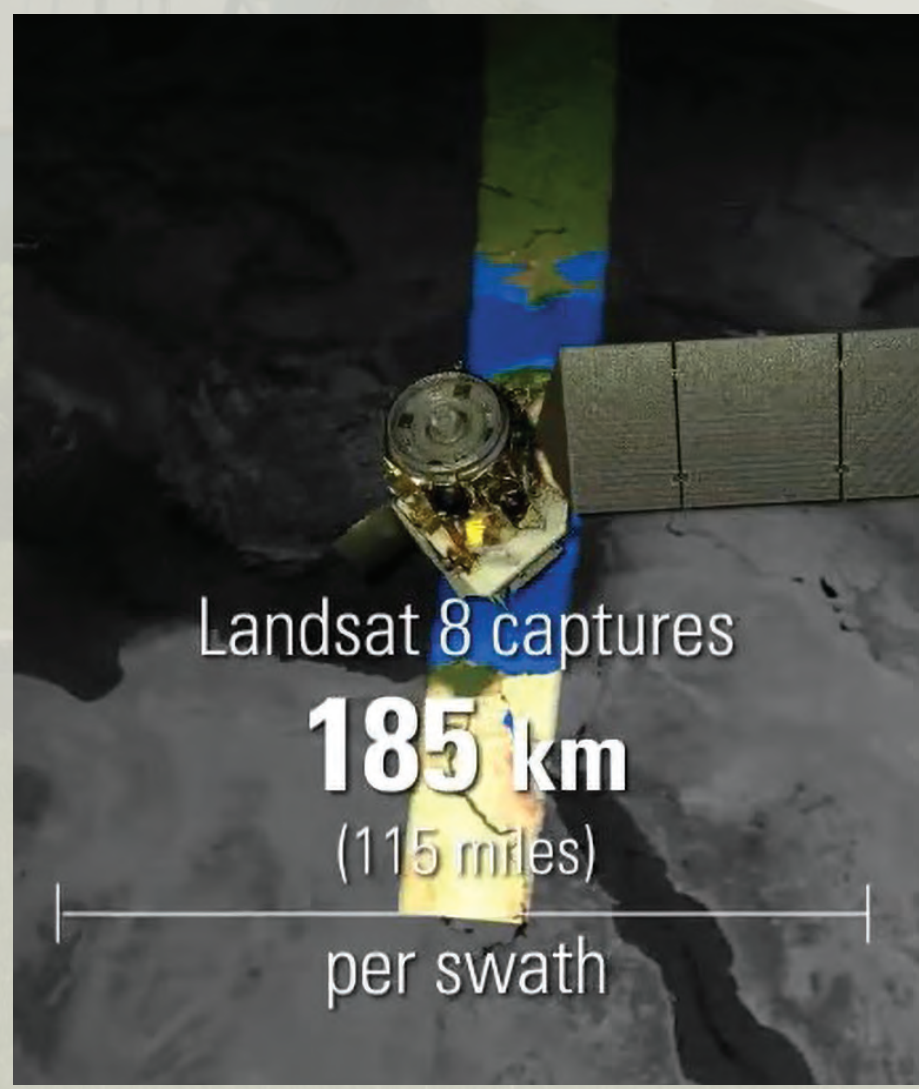


Science teams at EROS turn remote sensing data into reliable and actionable scientific datasets for the Nation and the world. Landsat's 30-meter resolution serves as the backbone for several land cover mapping efforts, each with differing uses:

- The National Land Cover Database (NLCD) sorts each 30-by-30-meter plot of land in the United States into a land cover class (such as deciduous forest, pasture/hay, or evergreen forest); and produces tree-canopy cover data layers, a change index, and a detailed impervious surface database useful for, studies of urban growth, the urban heat island effect, and runoff modeling.

- Land Change Monitoring, Assessment, and Projection (LCMAP) gleans its land cover and land change information from the depth of the Landsat archive, producing annual map layers from 1985 to 2020 that show not only basic land cover classes but also short-term disturbances, their intensity, and the approximate date that they occurred.

- Landscape Fire and Resource Management Planning Tools (LANDFIRE) offers 20 geospatial data layers outlining historical vegetation conditions, hundreds of vegetation types, vegetation height, fuels models, and more. LANDFIRE is valuable to the fire science community in planning for and responding to wildfires, as well as for a host of ecosystem studies. EROS serves as the mapping engine for the multiagency partnership.

Turning Landsat data into land cover mapping products, however, is only part of the EROS mission. Remote sensing researchers use satellite data and cloud computing resources to estimate evapotranspiration (ET) rates, or the rate of water loss through surface evaporation and transpiration from plants. ET estimates can be used to make wise use of resources in the United States and around the world as climate change and other factors put pressure on water supplies.

USGS EROS Center researchers Jeff Danielson (right) and Jeff Irwin (left) on site in Pohnpei, Micronesia.

\section{Data Center and Systems}

EROS was founded as a data center, and the Data Center and Systems Branch maintains that legacy by ensuring access to the highest-quality Earth observation data in an archive for more than 415,000 unique users annually. The archive houses nearly 9 million Landsat scenes, which are a major source for commercial applications such as Google Earth and Google Maps. This branch ensures access to Landsat data, as well as to derived Landsat products such as Landsat Surface Temperature, Landsat Burned Area, and Landsat Fractional Snow Cover. These data products allow scientists to more easily target and study specific types of change.

In addition to Landsat, the EROS archive hosts an array of other remotely sensed data - more than 1,100 different types, in fact. EROS has hosted NASA's Land Processes Distributed Active Archive Center (LP DAAC) for more than 30 years, providing online access to downloadable data. EROS has recently moved into cloud

data distribution. EROS uses the Amazon Web Services (AWS) Cloud to process and distribute data in collaboration with its on-premises information technology capabilities.

EROS is home to a Cray supercomputer called "Denali" - the largest system in the Department of the Interior (DOI) - and has been deemed a data center consolidation location for the USGS and DOI.

EROS is also the data hub for the Hazards Data Distribution System (HDDS), which ingests and makes available commercial and civilian remote sensing data collected after natural and manmade disasters such as hurricanes, wildfires, oil spills, and tsunamis. The data are available to first responders and researchers around the world and are useful in guiding emergency operations. EROS, in fact, is a member of the International Charter Space and Major Disasters, which focuses on disaster-related image distribution, often in remote parts of the world where access to satellite imagery is limited.

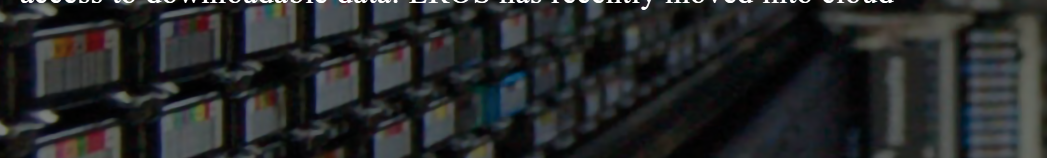




\section{New Missions}

Landsat satellites can operate for a long time. Landsat 5 even holds a world record for the longest-operating Earth observation satellite: 28 years and 10 months of service.

But EROS always has an eye to the future. The New Missions Branch collaborates with NASA on future Landsat missions, taking part in architecture study teams for observatory design and collaborating as new satellites are built and prepared for launch. NASA builds each Landsat satellite. After each launch and commissioning period, operations are handed over to the USGS. The most recent Landsat, Landsat 9, is set to launch in 2021, after which NASA, the USGS, and other agencies will work to prepare the orbiter to move into its operational status.

Even as preparations for Landsat 9 moved forward, discussions began about Landsat Next, the name given to the project that will produce the next generation of NASA/USGS collaboration. Whereas Landsat 9 is updated but similar to Landsat 8, which was launched in 2013, Landsat Next will aim to improve upon and add to the Landsat legacy by leveraging new technologies. Discussions about Landsat Next will contemplate the needs of the remote sensing science community as the number of satellite data sources rapidly proliferates in the commercial and civilian sectors, and the input from EROS will likely be key to assessing those needs.

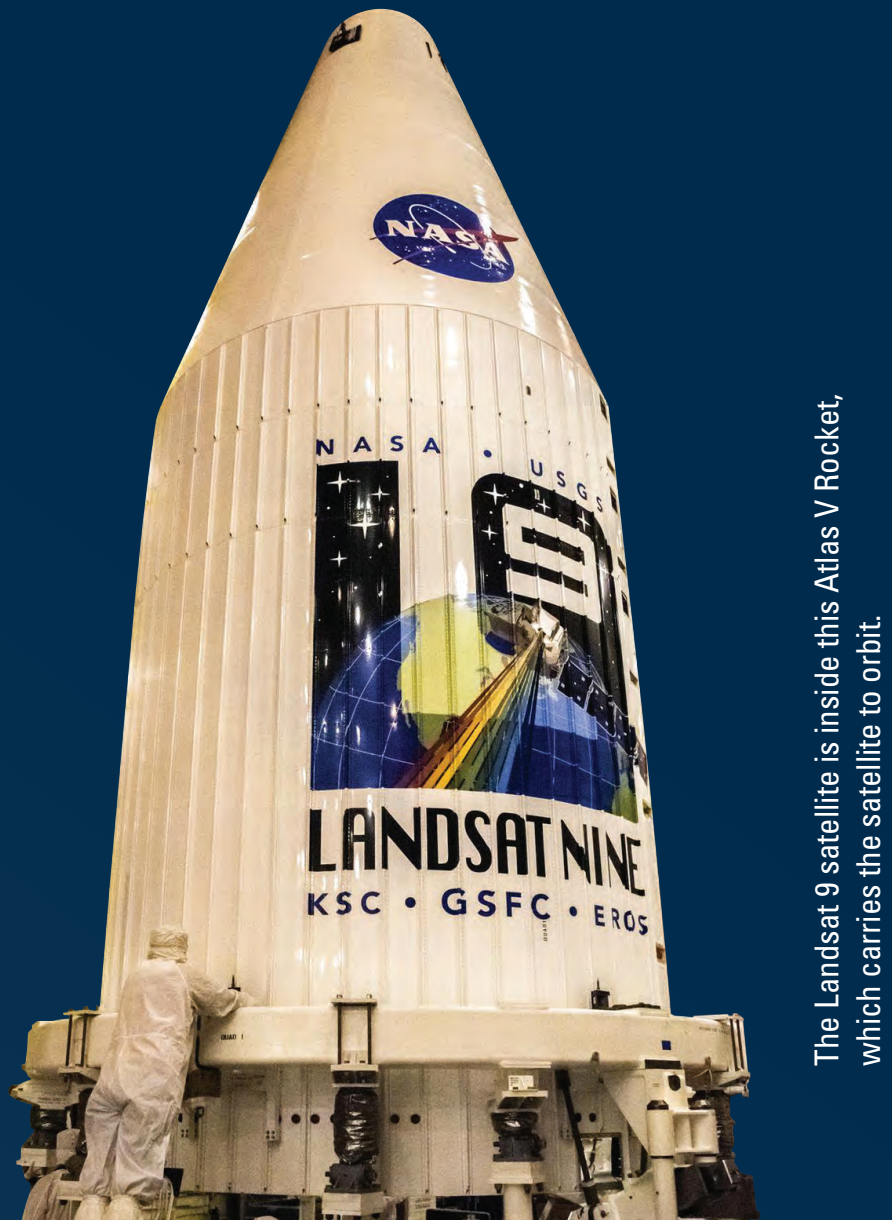

\section{Landsat Missions: Imaging the Earth Since 1972}

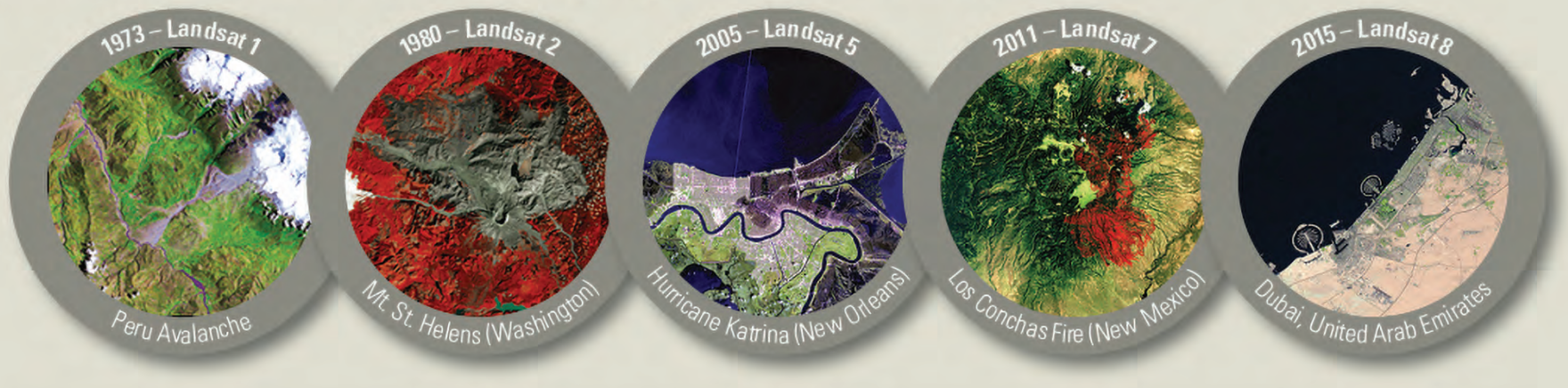

Landsat 1 July 1972 - January 1978

Landsat 2 January 1975 - July 1983

Landsat 3 March 1978 - September 1983

Landsat 4 July 1982 - December 1993

Landsat 6 October 1993

Landsat 7 April 1999 -

Landsat 8 February 2013 -

Landsat 92021

1970

$1975 \quad 1980$

$1985 \quad 1990$

1995

A goal of Landsat 9 is to maintain the Landsat Program's unbroken, decades-long record of repeat Earth observations. 


\section{Tours, Outreach, and Education}

The EROS Center is a short drive from Sioux Falls, South Dakota's largest city. The facility welcomes around 6,000 visitors in a typical year for its daily tours at 10 a.m. and 2 p.m., as well as additional walk-in visits and special group tours for $\mathrm{K}-12$ schools, universities, senior centers, and various other organizations.

Visitors can see a scale model of a Landsat satellite and the massive computer hardware wing where satellite data are stored, interact with digital displays that explain the use and value of remote sensing imagery, watch Landsat image collections in near real time, and more.

Outreach staff also visit Sioux Falls and the surrounding area for special events and small group talks, explaining the science behind Landsat and offering fun games like Landsat puzzles, guess the landscape feature brain teasers, and memory games. They are also available for classroom and community events by request.

Group tours of the EROS Center offer immersive, interactive experiences in geography and land change science for students and lifelong learners.

EROS is open to the public from 8 a.m. to 4 p.m., Monday through Friday, excluding Federal holidays:

47914 252nd St.

Sioux Falls, SD, 57198

605-594-6511

\section{Find Us Online!}

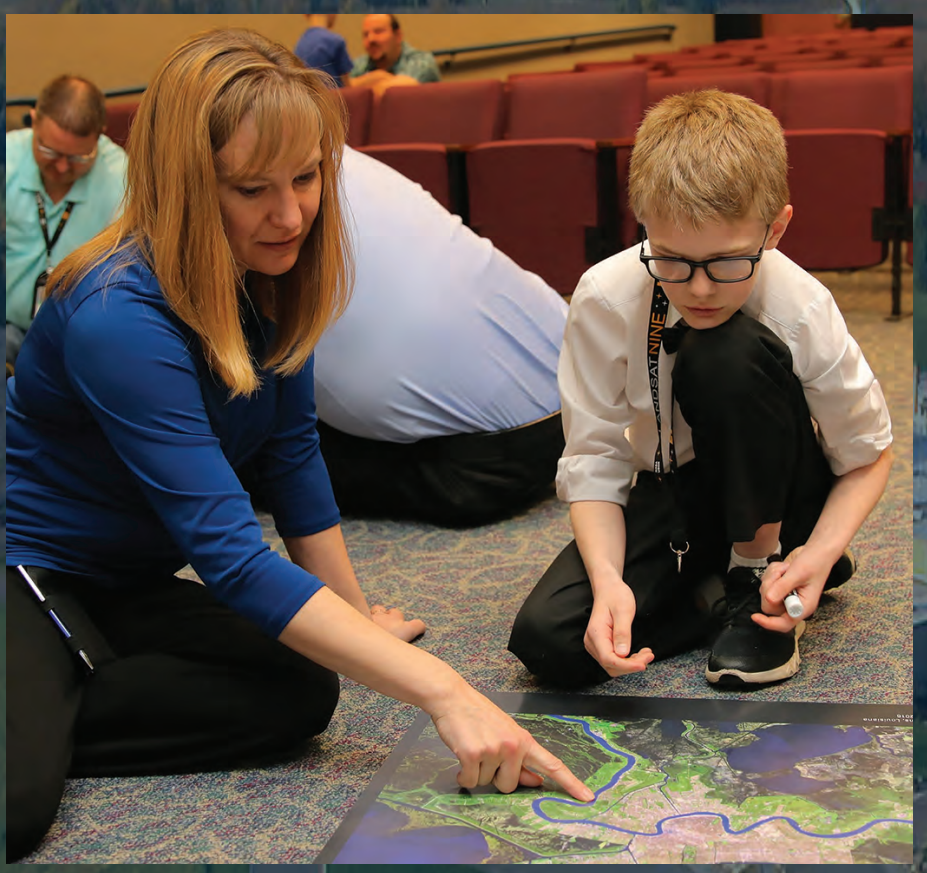

EROS outreach activities take place at the EROS Center, in classrooms, during community events, and online. To learn more and to access online learning resources, visit https://www.usgs.gov/centers/eros/outreach-and-education. 